\title{
Low Temperature Carbon Monoxide Sensor Based on Co(salen) Doped PEDOT: PSS
}

\author{
R. Memarzadeh ${ }^{1}$, S. Javadpour ${ }^{1}$, F. Panahi', Yoon-Bo Shim ${ }^{3}$ \\ ${ }^{1}$ Materials Science \& Eng. Department, Shiraz University, Shiraz, Iran \\ memarzadeh@shirazu.ac.ir \\ ${ }^{2}$ Chemistry Department, Science College, Shiraz University, Shiraz, Iran \\ ${ }^{3}$ Chemistry Department, Pusan National University, Busan, South Korea
}

\begin{abstract}
:
Poly (3, 4-ethylenedioxy thiophene) - poly (styrenesulfonate) (PEDOT:PSS) was used as a sensing matrix to fabricate a chemical carbon monoxide (CO) sensor. A Co(salen) complex was doped into the polymer matrix in various ratios to improve its response and selectivity to $\mathrm{CO}$ gas. The probe was examined to some common gases in the atmosphere, such as $\mathrm{O}_{2}, \mathrm{~N}_{2}$, and $\mathrm{CO}_{2}$ to evaluate its responsibility. This showed satisfactory response to $\mathrm{CO}$ which was different it from other gases. The response of the composite to $\mathrm{CO}$ was reversible (RSD $<5 \%$ ) change in resistance upon removal of $\mathrm{CO}$ gas from the test chamber. The doped polymer was characterized by Fourier Transform Infrared Spectroscopy (FT-IR) and UV- visuble spectroscopy (UV). Also, The effects of adding Co(salen) in the probe film on the response of the sensor were investigated by X-ray Photoelectron Spectroscopy (XPS). The dependence of the sensor response to the Co(salen) concentration was studied in the range of $0-1.5 \mathrm{wt} . \%$. The highest response was obtained at $1.0 \mathrm{wt} . \%$ Co(salen) in the composite $(25 \%$ $\pm 0.05)$.
\end{abstract}

Keywords: Chemical sensor, PEDOT:PSS, Co(salen), Carbon monoxide

\section{Introduction}

In recent years there have been many researches on making gas sensors based on conducting polymers (CPs). Some of these polymers are polyaniline, polypyrrole and polythiophene [1]. Due to easy preparation methods, unique properties, and stability in air, CPs have been applied to energy storage, memory devices, solar energy conversion, electrochromic display devices, batteries, light emitting cells and super capacitors.

A special application of CPs for detection of gaseous analytes belongs to well developed field of chemical sensors. Gases interacting with $\mathrm{CP}$ can be divided in two main classes: gases which chemically react with $\mathrm{CPs}$ and gases which physically absorb on CPs [2]. Composites of CP with metal or metal oxide nanoparticles and organic compounds provide new analytical possibilities. Because incorporation of these components in $\mathrm{CP}$ can enhance electron transfer through a direct or mediated mechanism with improved conductivity and enhanced stability.

Metal complexes such as phthalocyanines, porphyrins, ruthenium, palladium pyridine, and ferrocenyl derivative have been used in the past as functional groups in CPs for such applications [3].

Selectivity of the CP was controlled by the central metal atom. The cobalt compounds were used as a selective $\mathrm{CO}$ gas adsorbent to improve selectivity of the sensors [4].

In the present study, the sensor probe was fabricated by doping PEDOT: PSS with Co (salen) complex for increasing of response of the CP to carbon monoxide. PEDOT: PSS has a polar structure that shows a better discrimination of polar species such as water vapor, $\mathrm{NO}$ and $\mathrm{CO}$ gases. This study has been focused mainly on the characterization of PEDOT:PSS/Co(salen) composite and effect of incorporation of $\mathrm{Co}$ (salen) complex in to PEDOT:PSS on its chemical gas sensitivity.

\section{Experimental procedures:}

\subsection{Materials and interdigitate electrode}

Co(salen) complex was synthesized.Chemicals were purchased from Fluka and Aldrich (USA) 
and used without further purification. $\mathrm{CO}, \mathrm{N}_{2}$, $\mathrm{CO}_{2}$, and $\mathrm{O}_{2}$ gases were used as received from P.S.Chem Co. (USA). PEDOT:PSS was used as received from Aldrich Chemical Co. (USA), in the form of a blue colloidal suspension in water. This polymer suspension contained 1.0 wt $\%$ of solid content with a maximum viscosity of $15 \mathrm{mPa} \cdot \mathrm{s}$ and a surface resistance of $20 \mathrm{M} \Omega$. The Au-array electrode was produced by themicro-electromechanical systems (MEMS) technique consisting of $\mathrm{Au}$ interdigitate electrodes with an $\mathrm{Au}$ layer of $25.0 \mathrm{~nm}$ thickness and an effective area of $4.0 \times 5.0 \mathrm{~mm}$ deposited on the glass and the die size of 12.0 $\mathrm{mm} \times 11.0 \mathrm{~mm}$. The width of Au electrodes was $8.0 \mu \mathrm{m}$ and had equidistant gaps of $6.0 \mu \mathrm{m}$ between each electrode. The conducting polymer was spin coated on the interdigitate electrode.

\subsection{PreparationofCo(salen) doped PEDOT:PSS}

Different concentrations of Co(salen) were stirred into an aqueous solution of the polymer for several hours to prepare the Co(salen) doped PEDOT:PSS. During this process, PEDOT:PSS and the Co(salen) complex mixed together completely. The concentration of Co(salen) varied from 0.2 to $2.0 \mathrm{wt}$ \%. Spincoating was used to prepare a transparent thin film of doped PEDOT:PSS on interdigitated electrodes. A few drops of PEDOT:PSS containing Co(salen) was added to the base substrate, and the substrate was then spun at $3000 \mathrm{rpm}$ to produce the doped polymer film. The resulting sensor probes were exposed to different gases in a chamber, and the variation in resistance was monitored.

\section{Results and Discussion}

\subsection{Response of Co(salen) doped PEDOT:PSS to $\mathrm{CO}$}

Polymer films doped with different concentrations of $\mathrm{Co}$ (salen) were prepared on interdigitated $\mathrm{Au}$ electrodes and were then exposed to $\mathrm{CO}$ at $\mathrm{a}$ in the flow rate of 19.0 $\mathrm{ml} / \mathrm{min}$ at atmospheric pressure. Figure 1 shows the dependence of the Co(salen) concentration doped in the polymer on the sensor response to $\mathrm{CO}$ at room temperature. Sensor response is defined as the ratio of resistance measured in air $\left(R_{a}\right)$ to that measured in each gas $\left(R_{g}\right)$. A concentration of 1.0 wt. \% Co(salen) in the PEDOT:PSS thin film resulted in the maximum response to $\mathrm{CO}$. Increasing the Co(salen) concentration above 1.0 wt. $\%$ resulted in decreased response to co.

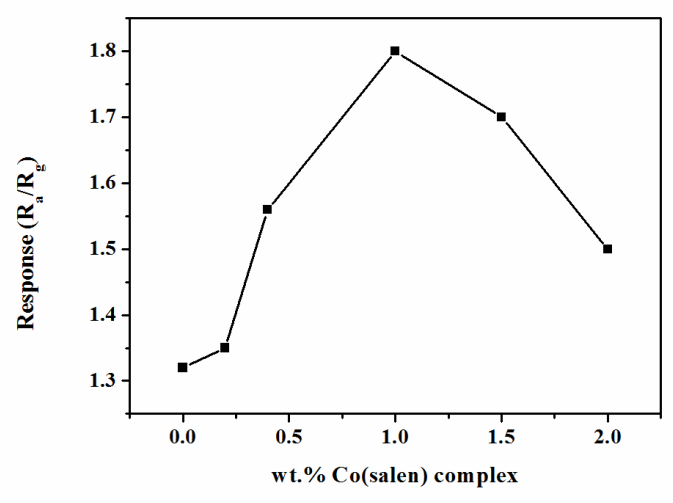

Figure 1, Response of Co(salen) doped PEDOT:PSS vs. Co(salen) concentration at room temperature.

\subsection{Selectivity of Co(salen) doped PEDOT:PSS to $\mathrm{CO}$}

The response of the PEDOT:PSS thin film doped with $1.0 \mathrm{wt}$. \% Co(salen) to $\mathrm{O}_{2}, \mathrm{~N}_{2}, \mathrm{CO}$, and $\mathrm{CO}_{2}$ was evaluated. Each gas was flowed in a vacuum chamber at a the rate of 17.0 $\mathrm{ml} / \mathrm{min}$. Figure 2 shows the percent variation in resistance of the sensing probe to the different gases tested. The sensor exhibited a better response to polar gases such as $\mathrm{CO}$ because PEDOT:PSS has a polar structure.

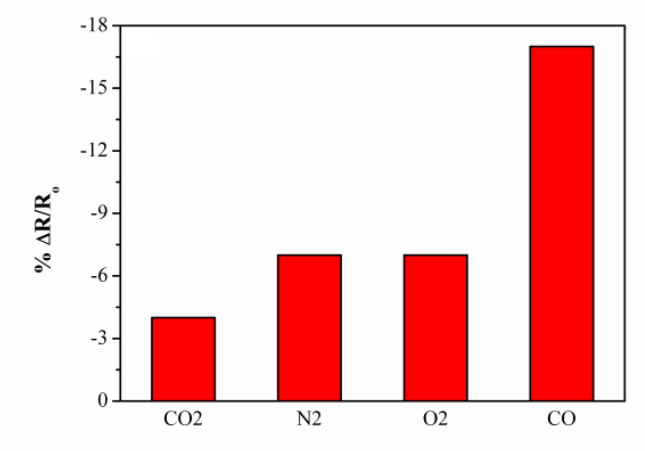

Figure 2 Response of the PEDOT:PSS/1.0 wt. \% $\mathrm{Co}$ (salen) composite to common gases in atmosphere.

However, there was a weak response to $\mathrm{O}_{2}$ and $\mathrm{CO}_{2}$. In addition, there was an unexpected decrease in the resistance of the sensor upon exposure to $\mathrm{N}_{2}$ gas. The desired vacuum pressure was not achieved completely in the testing chamber; therefore, it is possible that flowing nitrogen over the sensor removed trapped moisture on the surface of the sensor, explaining the drop in sensor resistance observed in a $\mathrm{N}_{2}$ atmosphere. Because the PEDOT:PSS has a polar structure, it can retain water moisture; contamination of the sensor by moisture in the atmosphere thus causes changes in its resistance. To mitigate this, a 
drying silicate was used to dry the inside of the testing chamber.

\subsection{UV-VIS Spectroscopy}

The UV-Vis absorption spectra of undoped, Co (salen) doped PEDOT: PSS thin film and Co(salen) complex (in ethanol solution) in the range 200-850 nm shown in Figure 3.

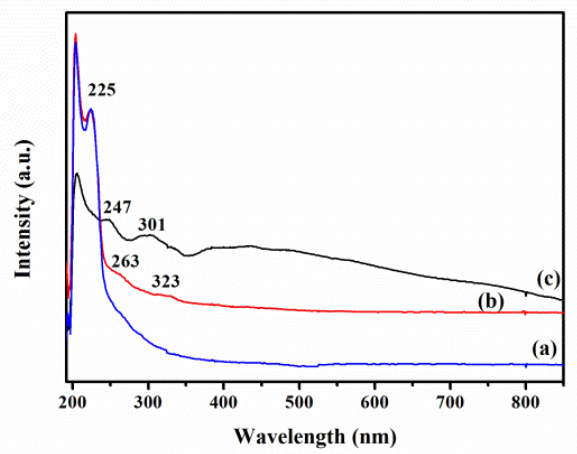

Figure 3 UV- visible spectra of (a) undoped, (b) Co (salen) doped PEDOT: PSS thin film and (c) Co(salen) complex.

The spectrum (a) exhibits an intense and sharp absorption peak at $225 \mathrm{~nm}$ that is related to PSS. Absorption peak of PEDOT is presence in the near-IR wavelength region around $3000 \mathrm{~nm}$ [6]. The UV-Vis. absorption spectrum of Co(salen) doped PEDOT:PSS thin film (spectrum (b)) is typical of PEDOT:PSS having an absorption maximum at $255 \mathrm{~nm}$. It can be seen that the addition of Co(salen) in a particular ratio (1wt.\% Co(salen)) has a remarkable effect on the absorption spectrum, by reducing the absorption and considerably shifting the absorption peak from 247 to 263 and from 301 to $323 \mathrm{~nm}$. The main characteristic band for the Co(salen) (spectrum (c)) is observed at around $300 \mathrm{~nm}$ which is assigned to the the $-\mathrm{C}=\mathrm{N}-$ group [7]. Therefore, The spectra confirm the incorporation of $\mathrm{Co}$ (salen) complex into the conducting polymer.

\subsection{FT-IR Analysis}

The Co (salen) doped PEDOT: PSS was confirmed by FTIR studies. The FTIR spectra are given in Figure 4. The curve (a), (b) and (c) correspond to the sample of pure PEDOT: PSS, pure Co (salen) and PEDOT: PSS/Co (salen) composite, respectively.

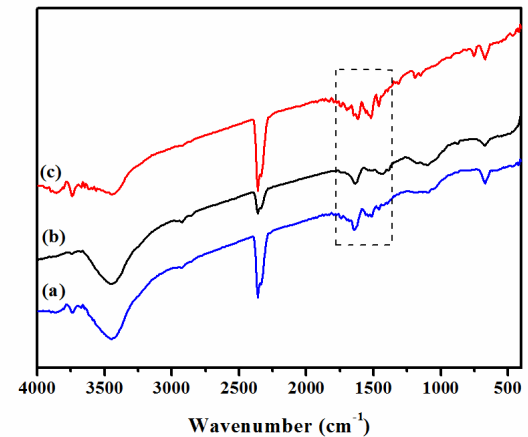

Figure 4 FTIR spectra of (a) PEDOT:PSS, (b)

Co(salen) comlex and (c) doped polymer.

Comparison between the FTIR spectra of the samples reveals some similar absorption bands in them. The peaks at around 1540 and 1640 $\mathrm{cm}^{-1}$ are related to the metallosalen and $\mathrm{C}=\mathrm{N}$ bonds which are present in PEDOT: PSS/Co (salen) composite [8]. The FTIR transmission spectrum of the PEDOT:PSS/Co(salen) shows bands at around 1504, 1389, 1088, 814, and $743 \mathrm{~cm}^{-1}$, which are presumably due to PEDOT:PSS bonds.

\subsection{XPS Analysis}

To investigate the interaction between Co(salen) and $\mathrm{CO}$ gas, XPS spectra were obtained for the Co(salen) doped PEDOT:PSS film before and after exposure to $\mathrm{CO}$ gas. Figure 5 shows the XPS spectra for (a) Co2p (b) S2p, (c) O1s, and (d) C1s in the sensor before (I) and after (II) reaction with $\mathrm{CO}$ gas.
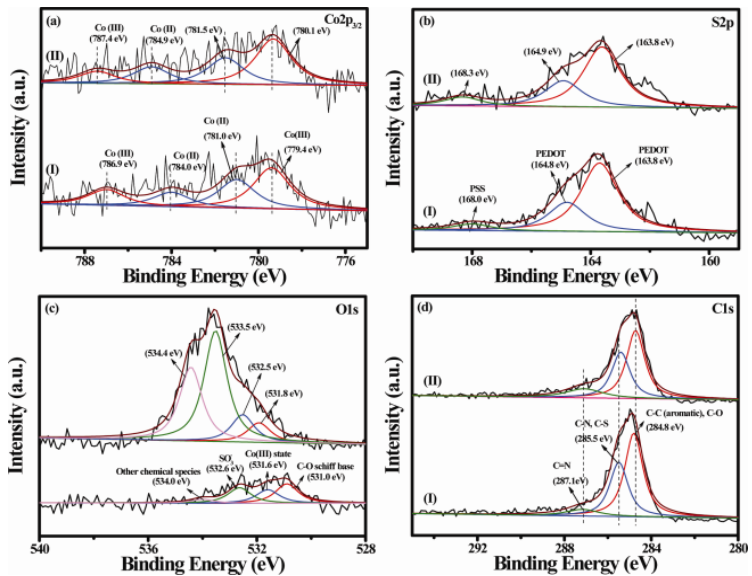

Figure 5 XPS spectra of (a) Co2p3/2, (b) $S 2 p$, (c) O1s, and (d) C1s for Co(salen)-doped PEDOT:PSS (I) before and (II) after the reaction with the CO gas.

The XPS spectrum of $C o 2 p_{3 / 2}$ before exposure to $\mathrm{CO}$ gas (Figure $5(\mathrm{a}(\mathrm{I}))$ ) contain two peaks at 779.4 and $781.0 \mathrm{eV}$, corresponding to the $\mathrm{Co}$ (III) and $\mathrm{Co}$ (II) ions, respectively [9]. Furthermore, additional satellite peaks were 
observed at approximately 784.0 and $786.9 \mathrm{eV}$ and are probably due to the Co (II) and Co (III) ions. Interestingly, a distinct peak shift from 781.0 to $781.5 \mathrm{eV}$ towards a higher binding energy was observed for the $\mathrm{Co}$ (III) and $\mathrm{Co}(\mathrm{II})$ ions after reaction with $\mathrm{CO}$ as shown in Figure $5(\mathrm{a}(\mathrm{II})))$, confirming the an interaction between the $\mathrm{Co}$ (salen) and $\mathrm{CO}$ gas. The $\mathrm{S} 2 \mathrm{p}$ spectrum exhibited peaks at 163.8 and $164.8 \mathrm{eV}$ corresponding to the C-S bond present in PEDOT. The spectrum showed an additional peak after doping of PSS at a higher binding energy of $168.0 \mathrm{eV}$, confirming successful doping of PSS on PEDOT (Figure $5(b(I))$ ) [10]. In a control experiment, however, we obtained XPS spectra for only PEDOT wherein the peak at $168.0 \mathrm{eV}$ was not found (data not shown). After reaction with $\mathrm{CO}$, distinct peak shifts from 168.0 to $168.3 \mathrm{eV}$ and from 164.8 to $164.9 \mathrm{eV}$ were observed due to the interaction between the polar groups of PEDOT:PSS and the CO gas (Figure $5(\mathrm{~b}(\mathrm{II}))$ ). This result again confirms an interaction between $\mathrm{CO}$ gas and the sensor. The 01s spectrum exhibited four peaks at 531.0, 531.6, 532.6, and 534.0 eV, corresponding to schiff base, Co(III) ion, sulfonate (PSS), and other chemical species, respectively (Figure 5(c(I))) [9]. Surprisingly, a peak shift with high intensity was observed after interaction of the sensor with $\mathrm{CO}$ gas (schiff base $(531.8 \mathrm{eV}), \mathrm{Co}(\mathrm{III})$ state $(532.5 \mathrm{eV})$, sulfonate $(533.5 \mathrm{eV})$, and other chemical species (534.4 eV)), as shown in Figure 5(c(II)). This reconfirms the successful interaction between the sensing probe and $\mathrm{CO}$ gas [11]. Of the observed peaks, the $\mathrm{SO}_{3}^{-}$peak exhibited the highest intensity due to water vapor adsorbed on the surface of sensing probe. Peaks at 284.8, 285.5, and 287.1 eV corresponded to C-C (Co(salen)), C-O (PEDOT), C-N (Co(salen)), C-S (PEDOT), and $\mathrm{C}=\mathrm{N}$ (Co(salen)), respectively. As seen in Figure $5(\mathrm{~d})$, there was no apparent change in the binding energy of $C$ for the doped polymer.

\section{Conclusion}

Co(salen) doped PEDOT:PSS acts as a sensing material for detection of $\mathrm{CO}$ gas under ambient conditions. The sensitivity of the PEDOT:PSS film to $\mathrm{CO}$ increased from $12 \pm$ 0.05 to $22 \pm 0.05 \%$ when a very low concentration of $\mathrm{Co}$ (salen) complex was added. The response of the Co(salen) doped PEDOT:PSS thin film to $\mathrm{CO}$ was quite fast and reversible. Further effort and research on this novel material may lead to a cost effective and high performance sensor for detection of $\mathrm{CO}$ poisoning.

\section{Acknowledgements}

This work was supported by the NRF grant funded by the MEST, South Korea (20100026167, 20100029128), and the international study program of Ministry of Science, Research and Technology of Iran.

\section{References}

[1] H. Bai, G. Shi, Gas sensors based on conducting polymers, Sensors 7, 267-307(2007).

[2] U.h Lange, N. V. Roznyatovskaya, V.r M. Mirsky Conducting polymers in chemical sensors and arrays, analytica chimica acta 614,1-26 (2008); doi:10.1016/j.aca.2008.02.068

[3] S. Paul, N.N. Chavan, S. Radhakrishnan, Polypyrrole functionalized with ferrocenyl derivative as a rapid carbon monoxide sensor, Synthetic Metals 159, 415-418 (2009); doi:10.1016/j.synthmet.2008.10.013

[4] H. J. Lee, J. H. Song, Y. S Yoon, T. S. Kim, K. J. Kim, W. K. Choi, Enhancement of CO Sensitivity of Indium Oxide-based semiconductor Gas Sensor Through Ultra-Thin Film Cobalt Adsorption, Sensors and actuators B 79, 200-205 (2001).

[5] F. Grillo, M. M. Natile, A. Glisenti, Low temperature oxidation of carbon monoxide: the influence of water and oxygen on the reactivity of a $\mathrm{Co} 3 \mathrm{O} 4$ powder surface, Applied Catalysis B: Environmental 48, 267-274(2004); doi:10.1016/j.apcatb.2003.11.003

[6] Chia-Yu Lin, Jian-Ging Chen, Chih-Wei Hu, James J. Tunney, Kuo-Chuan Ho, Using a PEDOT:PSS modified electrode for detecting nitric oxide gas, Sensors and Actuators B140, 402-406(2009); doi: 10.1016/j.snb.2009.04.041

[7] D. Wakizaka, T. Fushimi, H. Ohkita, S. Ito, Hole transport in conducting ultrathin films of PEDOT/PSS prepared by layer-by-layer deposition technique, Polymer 45, 8561-8565 (2004); doi: 10.1016/j.polymer.2004.10.007

[8] J. Połtowicz, K. Pamin , E. Tabor a, J. Haber , A. Adamski , Z. Sojka, Metallosalen complexes immobilized in zeolite $\mathrm{NaX}$ as catalysts of aerobic oxidation of cyclooctane, Applied Catalysis A: General,299,235242(2006);doi:10.1016/j.apcata. 2005.10.034,

[9] J. H. Burness, J. G. Dillard, L. T.Taylor, An X-Ray Photoelectron Spectroscopic Study of Cobalt(II) Schiff Base Complexes and Their Oxygenation Products, J. Am. Chem. Soc. 97, 6080-6088 (1975). DOI: 10.1021/ja00854a021

[10] X.Crispin, S.Marciniak, W. Osikowicz and et. al, Conductivity, Morphology, Interfacial Chemistry, and Stability of Poly(3,4-ethylene dioxythiophene) Poly(styrene sulfonate), J. Polym. Sci. Pol. Phys 41, 2561(2003).

[11] Y.Yang, Y. Jiang, J. Xu, J. Yu, Conducting PEDOT-PSS composite films assembled by LB technique, Colloids and Surfaces A 302, 157-161 (2007); doi:10.1016/j.colsurfa.2007.02.013 